\title{
Zero Singularities of Codimension Two and Three in Delay Differential Equations
}

\author{
Sue Ann Campbell * $\quad$ Yuan Yuan ${ }^{\dagger}$
}

September 10, 2008

\begin{abstract}
We give conditions under which a general class of delay differential equations has a point of Bogdanov-Takens or a triple zero bifurcation. We show how a centre manifold projection of the delay equations reduces the dynamics to a two or three dimensional systems of ordinary differential equations. We put these equations in normal form and determine how the coefficients of the normal forms depend on the original parameters in the model. Finally we apply our results to two neural models and compare the predictions of the theory with numerical bifurcation analysis of the full equations. One model involves a transcritical bifurcation, hence we derive and analyze the appropriate unfoldings for this case.
\end{abstract}

Mathematics Subject Classification: 92B20, 34K20, 34K15

Keywords: delay differential equations, centre manifold, normal form, Bogdanov-Takens bifurcation, triple zero singularity.

\section{Introduction}

The bifurcation analysis of codimension one and two linear singularities has been investigated by many researchers (e.g., see $[14,15,21]$ and references therein). By contrast, there are few studies of codimension three or higher problems in the literature. This is perhaps due to the relative rarity in ODE models of higher codimension singularities. In delay differential equations (DDEs), however, higher codimension singularities seem to occur more frequently. In particular, the Bogdanov-Taken singularity has been studied in many models with time

*Department of Applied Mathematics, University of Waterloo, Waterloo, Ontario, N2L 3G1, Canada

${ }^{\dagger}$ Department of Mathematics and Statistics, Memorial University of Newfoundland, St. John's NL A1C 5S7, Canada 
delays $[6,8,9,10,13,22,29,30,35,37,40,39]$ and the triple zero singularity shown to occur in a model for a delayed planar pendulum with $Z_{2}$ symmetry [31].

The purpose of this paper is to derive a mathematical framework for studying the doublezero (Bogdanov-Takens) and triple zero with geometric multiplicity one, in a large class of DDEs with two delays. Our goal is to determine conditions for the existence of these singularities and to analyze how the parameters of the DDE affect the secondary bifurcations which emanated from these points. While the Bogdanov-Takens has been studied in the context of general delay differential equations $[11,28]$ these studies have not looked at the specific questions we address. No general study of a DDE with a triple zero singularity has been done. Our approach will be to use the centre manifold theory for delay differential equations [17] to reduce the infinite dimensional dynamical system of the DDE to a finite dimensional system of ODEs and then apply the standard normal form reduction.

For completeness, we briefly review the literature on the triple zero singularity. All of these papers save one are related either to the derivation and analysis of the normal form/unfolding or its application to an ODE model. Medved [23] showed the triple zero singularity gives rise to saddle-node, Hopf, Bogdanov-Takens and Hopf-zero linear singularities, without characterizing them. Cushman and Sanders [3] and Iooss and Adelmeyer [20] derive normal forms for the triple zero singularity. Dumortier and Ibáñez [4] derive a normal form for all three parameter unfoldings of the triple zero singularity. Further classification and discussions are given in $[5,18]$ who consider the co-dimension four degeneracies when the lower order coefficients in the normal form vanish. Algaba et. al [1] study the triple zero normal form for a general three dimensional ODE and applied their results to the Rössler equation. Freire et al. [12] study the local codimension-two bifurcations, including Bogdanov-Takens and Hopf-zero, which arise in a three parameter unfolding of the triple zero singularity. Sieber and Krauskopf [31] show a model for a delayed planar pendulum with $Z_{2}$ symmetry has a triple zero singularity and derive the bifurcation diagram using the numerical continuation software DDE-BIFTOOL [7]. Ibáñez and Rodriguez [19] show that Shil'nikov homoclinic orbits occur in any generic unfolding of the triple zero singularity.

The plan for the article is as follows. In section 2 we review some background we will 
use for our analysis, derive the centre manifold of a general DDE with Bogdanov-Takens or triple zero singularity with index one and then state the normal forms for these singularities. In section 3 we apply these results to the following system of DDE's:

$$
\begin{aligned}
& \dot{x}_{1}(t)=f_{1}\left(x_{1}(t), x_{2}(t)\right)+g_{1}\left(x_{1}\left(t-\tau_{s}\right), x_{2}(t-\tau)\right), \\
& \dot{x}_{2}(t)=f_{2}\left(x_{1}(t), x_{2}(t)\right)+g_{2}\left(x_{1}(t-\tau), x_{2}\left(t-\tau_{s}\right)\right) .
\end{aligned}
$$

In particular, we determine how the coefficients of the normal form depend on the parameters of the DDE. In section 4 we consider two specific models which fall into this class: an additive neural network studied by Shayer and Campbell [30] and a model for a delayed recurrent neural circuit similar to one studied by Plant [27]. We use the results of section 3 to determine, for each model, conditions on the parameters such that a Bogdanov-Takens singularity occurs. We show that the neural network model does not possess a triple zero point, while the recurrent circuit model can, for appropriate choice of parameters. We then apply the results of section 3 to determine the normal forms at the critical parameter values for these systems. Finally, we state the unfoldings of these normal forms, analyze their behaviour and compare the predictions of the theory with numerical studies of the full, nonlinear DDEs using the numerical continuation software DDE-BIFTOOL [7]. Conclusions are given in section 5 .

\section{General Approach}

Define $\mathbf{u}_{t}(\theta)=\mathbf{u}(t+\theta),-h \leq \theta \leq 0, \mathcal{C}=C\left([-h, 0], \mathbb{R}^{n}\right)$ and consider delay differential equation

$$
\dot{\mathbf{u}}(t)=\mathbf{F}\left(\mathbf{u}_{t}, \alpha\right)
$$

where $\mathbf{u} \in \mathbb{R}^{n}, \mathbf{F} \in C^{r}\left(\mathcal{C}, \mathbb{R}^{n}\right), r>1$ and $\alpha \in \mathbb{R}^{m}$ is a parameter.

An equilibrium solution of equation (1) is a solution $\mathbf{u}_{t}(\theta)=\mathbf{u}^{*},-h \leq \theta \leq 0$. We assume that the system possesses an equilibrium solution, i.e. that there exists $\mathbf{u}^{*} \in \mathbb{R}^{n}$ such that $\mathbf{F}\left(\mathbf{u}^{*}, \alpha\right)=0$.

Linearizing equation (1) about this equilibrium yields

$$
\dot{\mathbf{x}}=\mathcal{L}\left(\mathbf{x}_{t}\right)
$$


where $\mathbf{x}_{t}=\mathbf{u}_{t}-\mathbf{u}^{*}$ and $\mathcal{L}$ is a linear operator which may be represented

$$
\mathcal{L} \mathbf{x}_{t}=\int_{-h}^{0} d \eta(\theta) \mathbf{x}(t+\theta)
$$

with $\eta(\theta)$ a function of bounded variation.

\subsection{Results for Linear Equations}

We begin by relating some results concerning the solutions of the linear delay equation (2). This discussion follows the book of Hale and Lunel [17, Chapter 7].

The local stability of the equilibrium may be studied by considering the eigenvalues of the linear operator $\mathcal{L}$ in the equation above. It can be shown that the eigenvalues, $\lambda$, are the roots of the characteristic equation

$$
p(\lambda)=\operatorname{det} \Delta(\lambda)=0
$$

where

$$
\Delta(\lambda)=\lambda I-\int_{-h}^{0} e^{\lambda \theta} d \eta(\theta)
$$

and $I$ is the $n \times n$ identity matrix.

A point of Bogdanov-Takens (BT) bifurcation of the fixed point $\mathbf{u}^{*}$ (also called a double zero singularity) occurs if the following conditions are satisfied.

(i) The characteristic equation has a zero root of multiplicity two, i.e.,

$$
p(0)=0, p^{\prime}(0)=0, p^{\prime \prime}(0) \neq 0
$$

(ii) No other root of the characteristic equation has zero real part, i.e.,

$$
p(i \omega) \neq 0
$$

for any $\omega \in \mathbb{R}$.

If the characteristic equation has a zero root of multiplicity three, which means

$$
p(0)=p^{\prime}(0)=p^{\prime \prime}(0)=0 \text { and } p^{\prime \prime \prime}(0) \neq 0,
$$

and the condition (6) holds, this gives rise to a triple zero singularity. 


\subsection{Centre Manifold Analysis}

Consider the original nonlinear DDE (1). If $\mathbf{F}$ is sufficiently smooth, one can expand the right side of the equation in a Taylor series about the equilibrium solution $\mathbf{u}^{*}$ :

$$
\dot{\mathbf{x}}=\mathcal{L} \mathbf{x}_{t}+\mathbf{G}\left(\mathbf{x}_{t}\right)
$$

where $\mathbf{x}_{t}, \mathcal{L}$ are as above and $\mathbf{G}: \mathcal{C} \rightarrow \mathbb{R}^{n}$ satisfies $G(0)=D G(0)=0$.

Under the conditions given above ((5) and (6), or (6) and (7)), it can be shown [17] that the space of solutions, $\mathcal{C}$, can be decomposed as $\mathcal{C}=P \oplus Q$. Here $P$ is an $m$-dimensional ( $m=2$ or 3 ) subspace spanned by the solutions of (2) corresponding to the $m$ zero real part eigenvalues (sometimes called the centre eigenspace), $Q$ is the complementary space, and $P$ and $Q$ are invariant under the flow associated with (2). Further, for the nonlinear equation (8), there exists a centre manifold in $\mathcal{C}$ which is a finite $(m=2$ or 3$)$ dimensional, invariant manifold. We denote the centre manifold by

$$
M_{f}=\left\{\phi \in \mathcal{C}: \phi=\Phi(\theta) \mathbf{z}+g(\mathbf{z}, \theta), \mathbf{z} \text { in a neighbourhood of zero in } \mathbb{R}^{m}\right\},
$$

where $\mathbf{\Phi}=\left[\phi_{1}, \ldots, \phi_{m}\right]$ is a basis for the subspace $P$ and $g$ is a function in the subspace $Q$ which is $O\left(\|\mathbf{z}\|^{2}\right)$.

If all eigenvalues of the characteristic equation (3) with nonzero real part have negative real part, then the center manifold will be attracting and the long term behaviour of solutions to the nonlinear equation (8) is well approximated by the flow on this manifold. This flow is given by

$$
\mathbf{x}_{t}(\theta)=\boldsymbol{\Phi}(\theta) \mathbf{z}(t)+g(\mathbf{z}(t), \theta),
$$

where $\mathbf{z} \in \mathbb{R}^{m}$ satisfies the ordinary differential equation $[16,36]$

$$
\dot{\mathbf{z}}=B \mathbf{z}+\mathbf{b G}(\mathbf{\Phi} \mathbf{z}+g(\mathbf{z}, \theta))
$$

$\Phi^{\prime}(\theta)=\boldsymbol{\Phi}(\theta) B, B$ is a $m \times m$ constant matrix with $m$ zero eigenvalues, and $\mathbf{b}$ is determined as described below.

Consider the equation adjoint to Eq.(2). It also has $m$ zero eigenvalues. Let $\Psi=$ $\left[\psi_{1}, \ldots, \psi_{m}\right]^{T}$ be a basis for the invariant subspace spanned by the solutions of the adjoint 
equation corresponding to these eigenvalues. Then $\mathbf{b}=\Psi(0)$. The basic functional form (up to arbitrary constants) of $\Psi$ can be found by noting that it must satisfy $-\Psi^{\prime}(s)=B \Psi[11,36]$. The integration constants may be specified by requiring that $\Psi$ satisfy the normalization condition

$$
\langle\Psi, \Phi\rangle=\mathrm{I}
$$

where $\mathrm{I}$ is the $m \times m$ identity matrix, $\langle\Psi, \Phi\rangle_{i j}=\left\langle\psi_{i}, \phi_{j}\right\rangle$ and

$$
<\psi, \phi>=\psi(0) \phi(0)-\int_{-h}^{0} \int_{0}^{\theta} \psi(\xi-\theta) d \eta(\theta) \phi(\xi) d \xi
$$

is the bilinear form associated with Eq. (2). In section 3 we shall consider a system with only discrete delays. In this case, the components of the matrix valued function $\eta(\theta)$ will be sums of step functions at the delay values.

In summary, the long-term behaviour of solutions of the DDE (1) in a neighbourhood of the equilibrium solution $\mathbf{u}^{*}$ can be described if we can understand the behaviour of the solutions of a finite dimensional system of ordinary differential equations (10). To investigate this system of ODE's further we now consider how to put it in its simplest form.

To be more precise, for the BT singularity, $P$ is a two dimensional subspace with a basis constructed as follows. Consider the linear equation on $\mathbb{R}^{n}$

$$
\Delta(0) \mathbf{v}=0
$$

From conditions (5) it is clear that a nontrivial solution, $\mathbf{v}_{1}$, of this equation exists. In the case of a BT singularity, the geometric multiplicity of the eigenvalue 0 is 1 , implying the solution space of (12) is one-dimensional. In this situation, a basis for $P$ is given by [17, Chapter 7, Theorem 4.2]

$$
\mathbf{\Phi}=\left[\phi_{1}, \phi_{2}\right]=\left[\mathbf{v}_{1}, \mathbf{v}_{2}+\theta \mathbf{v}_{1}\right]
$$

where $\mathbf{v}_{2}$ is a solution of the linear equation

$$
\Delta(0) \mathbf{v}+\Delta^{\prime}(0) \mathbf{v}_{1}=0
$$

Here $\Delta^{\prime}(0)=\left.\Delta^{\prime}(\lambda)\right|_{\lambda=0}$ is the derivative of the matrix function $\Delta(\lambda)$ with respect to $\lambda$ at $\lambda=0$. 
For the triple zero singularity, $P$ is a three-dimensional invariant subspace. We will focus on the case where the solution space of (12) is one-dimensional. Let $\mathbf{v}_{2}$ and $\mathbf{v}_{3}$ be the solutions of the linear equations

$$
\begin{aligned}
\Delta^{\prime}(0) \mathbf{v}_{1}+\Delta(0) \mathbf{v}_{2} & =0 \\
\frac{1}{2} \Delta^{\prime \prime}(0) \mathbf{v}_{1}+\Delta^{\prime}(0) \mathbf{v}_{2}+\Delta(0) \mathbf{v}_{3} & =0
\end{aligned}
$$

where $\Delta^{\prime \prime}(0)=\left.\Delta^{\prime \prime}(\lambda)\right|_{\lambda=0}$ is the second derivative of the matrix function $\Delta(\lambda)$ with respect to $\lambda$ at $\lambda=0$. Then the basis for $P$ can be chosen as [17, Chapter 7 , Theorem 4.2]

$$
\mathbf{\Phi}=\left[\phi_{1}, \phi_{2}, \phi_{3}\right]=\left[\mathbf{v}_{1}, \mathbf{v}_{2}+\theta \mathbf{v}_{1}, \mathbf{v}_{3}+\mathbf{v}_{2} \theta+\mathbf{v}_{1} \frac{\theta^{2}}{2}\right]
$$

\subsection{Normal Form Reduction}

When only the lowest order terms in the normal forms are needed, the centre manifold function, $g$, is not needed to calculate the normal form. To see this, rewrite (10) as

$$
\dot{\mathbf{z}}=B \mathbf{z}+\Psi(0)\left[G_{2}(\Phi \mathbf{z}+g(z, \theta))+G_{3}(\Phi \mathbf{z}+g(z, \theta))+\text { h.o.t. }\right]
$$

where $G_{j}$ represents the terms of order $j$ in $G$. Expanding each $G_{j}$ in a Taylor series about $\Phi \mathbf{z}$ yields

$$
\dot{\mathbf{z}}=B \mathbf{z}+\Psi(0) G_{2}(\Phi \mathbf{z})+\text { h.o.t. }
$$

where the higher order terms are cubic in $\mathbf{z}$ and thus will play no role in the calculation of the quadratic terms of the normal form.

The near identity transformation

$$
\mathbf{z}=\mathbf{w}+\hat{\mathbf{F}}_{2}
$$

for appropriate choice of $\hat{\mathbf{F}}$, may then be used to bring system (17) into a simpler (normal) form. For BT singularity, this form is (see $[2,11]$ or $[14$, Section 7.3])

$$
\dot{\mathbf{w}}=B_{1} \mathbf{w}+A_{20}\left(\begin{array}{l}
0 \\
w_{1}^{2}
\end{array}\right)+A_{11}\left(\begin{array}{l}
0 \\
w_{1} w_{2}
\end{array}\right)+\text { h.o.t. }
$$

For triple zero singularity, the normal form is (see [38])

$$
\dot{\mathbf{w}}=B_{2} \mathbf{w}+A_{200}\left(\begin{array}{l}
0 \\
0 \\
w_{1}^{2}
\end{array}\right)+A_{110}\left(\begin{array}{l}
0 \\
0 \\
w_{1} w_{2}
\end{array}\right)+A_{101}\left(\begin{array}{l}
0 \\
0 \\
w_{1} w_{3}
\end{array}\right)+A_{020}\left(\begin{array}{l}
0 \\
0 \\
w_{2}^{2}
\end{array}\right)+\text { h.o.t. . }
$$


Here

$$
B_{1}=\left[\begin{array}{ll}
0 & 1 \\
0 & 0
\end{array}\right] \quad B_{2}=\left[\begin{array}{lll}
0 & 1 & 0 \\
0 & 0 & 1 \\
0 & 0 & 0
\end{array}\right]
$$

and all the coefficients $A_{i j}$ or $A_{i j k}$ can been expressed in terms of the coefficients in (17).

We note that when $\mathbf{F}$ is an odd function of $\mathbf{x}_{t}$ then $\mathbf{G}$ will include terms with odd powers of $\mathbf{x}_{t}$ and the coefficients $A_{i j}\left(A_{i j k}\right)$ at the second order in Eqs. $(18)((19))$ will be zero. Then we have to calculate the normal form up to third order. For the BT singularity, this is (see [2] or [14, Section 7.3])

$$
\dot{\mathbf{w}}=B_{1} \mathbf{w}+A_{30}\left(\begin{array}{l}
0 \\
w_{1}^{3}
\end{array}\right)+A_{21}\left(\begin{array}{l}
0 \\
w_{1}^{2} w_{2}
\end{array}\right)+\text { h.o.t. }
$$

whereas for triple zero singularity it is (see [38])

$$
\begin{aligned}
\dot{\mathbf{w}}=B_{2} \mathbf{w} & +A_{300}\left(\begin{array}{l}
0 \\
0 \\
w_{1}^{3}
\end{array}\right)+A_{210}\left(\begin{array}{l}
0 \\
0 \\
w_{1}^{2} w_{2}
\end{array}\right)+A_{120}\left(\begin{array}{l}
0 \\
0 \\
w_{1} w_{2}^{2}
\end{array}\right) \\
& +A_{030}\left(\begin{array}{l}
0 \\
0 \\
w_{2}^{3}
\end{array}\right)+A_{201}\left(\begin{array}{l}
0 \\
0 \\
w_{1}^{2} w_{3}
\end{array}\right)+A_{102}\left(\begin{array}{l}
0 \\
0 \\
w_{1} w_{3}^{2}
\end{array}\right)+\text { h.o.t. }
\end{aligned}
$$

\section{A general two-dimensional system}

We consider the system

$$
\begin{aligned}
& \dot{x}_{1}(t)=f_{1}\left(x_{1}(t), x_{2}(t)\right)+g_{1}\left(x_{1}\left(t-\tau_{s}\right), x_{2}(t-\tau)\right), \\
& \dot{x}_{2}(t)=f_{2}\left(x_{1}(t), x_{2}(t)\right)+g_{2}\left(x_{1}(t-\tau), x_{2}\left(t-\tau_{s}\right)\right) .
\end{aligned}
$$

Note that this includes many artificial neural network models [9, 10, 30], gene regulatory network models [34], various models for metal cutting [32, 33], car-following models [25], and models for nonlinear oscillators with delayed feedback [27, 31].

We assume that the equation has an equilibrium solution $\mathbf{x}^{*}=\left(x_{1}^{*}, x_{2}^{*}\right)$, i.e. $f_{i}\left(x_{1}^{*}, x_{2}^{*}\right)=$ $g_{i}\left(x_{1}^{*}, x_{2}^{*}\right)=0$, and that $f_{i}, g_{i} \in C^{3}, i=1,2$. We write the Taylor expansion about $\mathbf{x}^{*}$ of the functions $g_{i}, f_{i}$ up to $m^{\text {th }}$ order terms $(m=2$ or 3$)$ as

$$
f_{i}\left(x_{1}(t), x_{2}(t)\right)=\sum_{n=1}^{m} \sum_{j+k=n} a_{i j k} x_{1}^{j}(t) x_{2}^{k}(t),
$$




$$
\begin{aligned}
& g_{1}\left(x_{1}\left(t-\tau_{s}\right), x_{2}(t-\tau)\right)=\sum_{n=1}^{m} \sum_{j+k=n} b_{1 j k} x_{1}^{j}\left(t-\tau_{s}\right) x_{2}^{k}(t-\tau), \\
& g_{2}\left(x_{1}(t-\tau), x_{2}\left(t-\tau_{s}\right)\right)=\sum_{n=1}^{m} \sum_{j+k=n} b_{2 j k} x_{1}^{j}(t-\tau) x_{2}^{k}\left(t-\tau_{s}\right) .
\end{aligned}
$$

The linearization of $(22)$ about $\mathrm{x}^{*}$ is then

$$
\begin{aligned}
& \dot{u}_{1}(t)=a_{110} u_{1}(t)+a_{101} u_{2}(t)+b_{110} u_{1}\left(t-\tau_{s}\right)+b_{101} u_{2}(t-\tau), \\
& \dot{u}_{2}(t)=a_{210} u_{1}(t)+a_{201} u_{2}(t)+b_{210} u_{1}(t-\tau)+b_{201} u_{2}\left(t-\tau_{s}\right) .
\end{aligned}
$$

It follows that the characteristic matrix is

$$
\Delta(\lambda)=\left[\begin{array}{ll}
\lambda-a_{110}-b_{110} e^{-\tau_{s} \lambda} & -a_{101}-b_{101} e^{-\tau \lambda} \\
-a_{210}-b_{210} e^{-\tau \lambda} & \lambda-a_{201}-b_{201} e^{-\tau_{s} \lambda}
\end{array}\right],
$$

and that the characteristic equation is

$p(\lambda)=\operatorname{det} \Delta(\lambda)=\left(\lambda-a_{110}-b_{110} e^{-\tau_{s} \lambda}\right)\left(\lambda-a_{201}-b_{201} e^{-\tau_{s} \lambda}\right)-\left(a_{101}+b_{101} e^{-\tau \lambda}\right)\left(a_{210}+b_{210} e^{-\tau \lambda}\right)$.

The nonlinear part of system (22), to order $m$, has the following form

$$
G_{m}\left(\begin{array}{c}
\varphi_{1} \\
\varphi_{2}
\end{array}\right)=\left(\begin{array}{l}
\sum_{n=2}^{m} \sum_{j+k=n} a_{1 j k} \varphi_{1}^{j}(0) \varphi_{2}^{k}(0)+\sum_{n=2}^{m} \sum_{j+k=n} b_{1 j k} \varphi_{1}^{j}\left(\tau_{s}\right) \varphi_{2}^{k}(-\tau) \\
\sum_{n=2}^{m} \sum_{j+k=n} a_{2 j k} \varphi_{1}^{j}(0) \varphi_{2}^{k}(0)+\sum_{n=2}^{m} \sum_{j+k=n} b_{2 j k} \varphi_{1}^{j}(\tau) \varphi_{2}^{k}\left(-\tau_{s}\right)
\end{array}\right) .
$$

In this paper, we focus on two singularity cases related to the existence of zero roots, with multiplicity 2 or 3 , of the characteristic equation (25).

\subsection{Bogdanov-Takens Singularity}

From eq. (5), a Bogdanov-Takens singularity can occur in (22) if the following conditions are satisfied

$$
\begin{aligned}
\left(a_{110}+b_{110}\right)\left(a_{201}+b_{201}\right)-\left(a_{101}+b_{101}\right)\left(a_{210}+b_{210}\right) & =0 \\
-\left(1+\tau_{s} b_{110}\right)\left(a_{201}+b_{201}\right)-\left(1+\tau_{s} b_{201}\right)\left(a_{110}+b_{110}\right) & \\
+\tau\left[b_{101}\left(a_{210}+b_{210}\right)+b_{210}\left(a_{101}+b_{101}\right)\right] & =0,
\end{aligned}
$$


and $p^{\prime \prime}(0) \neq 0$, i.e.,

$$
\tau_{s}^{2}\left(a_{201} b_{110}+4 b_{110} b_{201}+a_{110} b_{201}\right)+2 \tau_{s}\left(b_{201}+b_{110}\right)-\tau^{2}\left(a_{210} b_{101}+4 b_{101} b_{210}+a_{101} b_{210}\right)+2 \neq 0 .
$$

As discussed above, the invariant subspace $P$ corresponding to the zero roots is two dimensional with basis $\Phi_{1}=\left[\mathbf{v}_{1}, \mathbf{v}_{2}+\theta \mathbf{v}_{1}\right]$. For convenience, we choose

$$
\mathbf{v}_{1}=\left(\begin{array}{c}
1 \\
m_{1}
\end{array}\right), \quad \mathbf{v}_{2}=\left(\begin{array}{c}
0 \\
m_{2}
\end{array}\right)
$$

where $m_{1}=-\frac{a_{110}+b_{110}}{a_{101}+b_{101}}, m_{2}=\frac{1+\tau_{s} b_{110}+\tau b_{101} m_{1}}{a_{101}+b_{101}}$.

The corresponding basis, $\Psi_{1}$, for the adjoint problem must have the form

$$
\Psi_{1}(s)=\left[\begin{array}{cc}
-d_{1} s+d_{3} & -d_{2} s+d_{4} \\
d_{1} & d_{2}
\end{array}\right]
$$

to satisfy $-\Psi_{1}^{\prime}=B_{1} \Psi_{1}$. Using $\left\langle\Psi_{1}, \Phi_{1}\right\rangle=I$ we can determine the constants $d_{i}(i=$ $1,2,3,4)$. We omit the expressions for the $d_{i}$ here due to their length, however, they are given for the examples in the next section. Note that this gives $\Psi_{1}(0)=\left[\begin{array}{ll}d_{3} & d_{4} \\ d_{1} & d_{2}\end{array}\right]$.

To this point, we have reduced the original infinite dimensional DDE system to a twodimensional ODE system

$$
\dot{\mathbf{z}}=B_{1} \mathbf{z}+\Psi_{1}(0) G_{2}\left(\Phi_{1} z\right)+\text { h.o.t. }
$$

where $\mathbf{z}=\left(\begin{array}{c}z_{1} \\ z_{2}\end{array}\right)$. As discussed in section 2.3 (see eq. (18)), a normal form to second order for this equation is given by

$$
\begin{aligned}
& \dot{w}_{1}=w_{2} \\
& \dot{w}_{2}=A_{20} w_{1}^{2}+A_{11} w_{1} w_{2}+\text { h.o.t. }
\end{aligned}
$$

To find the relationship between the coefficients of (30) and (31) we need to know the form of the near-identity transformation that relates them. This transformation is given in [11]. Using this transformation and $\boldsymbol{\Phi}_{\mathbf{1}} \mathbf{z}=\left(\begin{array}{c}z_{1}+\theta z_{2} \\ m_{1} z_{1}+\left(m_{2}+\theta m_{1}\right) z_{2}\end{array}\right)$, we find

$$
\begin{aligned}
& A_{20}=d_{1} E_{1}+d_{2} E_{2} \\
& A_{11}=d_{1} T_{1}^{1}\left(\tau_{s}, \tau\right)+d_{2} T_{1}^{2}\left(\tau, \tau_{s}\right)+2\left(d_{3} E_{1}+d_{4} E_{2}\right),
\end{aligned}
$$


where

$$
\begin{aligned}
& E_{i}= a_{i 20}+a_{i 11} m_{1}+a_{i 02} m_{1}^{2}+b_{i 20}+b_{i 11} m_{1}+b_{i 02} m_{1}^{2}=\sum_{j+k=2}\left(a_{i j k}+b_{i j k}\right) m_{1}^{k}, \\
& T_{1}^{i}\left(\tau_{s}, \tau\right)= a_{i 11} m_{2}+2 a_{i 02} m_{1} m_{2} \\
&+2 b_{i 20} n_{1}\left(\tau_{s}\right)+b_{i 11}\left[m_{1} n_{1}\left(\tau_{s}\right)+n_{2}(\tau)\right]+2 b_{i 02} m_{1} n_{2}(\tau) \\
&\left(i=1,2, \quad n_{1}\left(\tau_{s}\right)=-\tau_{s}, \quad n_{2}(\tau)=m_{2}-\tau m_{1}\right)
\end{aligned}
$$

In the following, we denote $n_{1}\left(\tau_{s}\right), n_{2}(\tau)$ by $n_{1}, n_{2}$, and $T_{1}^{1}\left(\tau_{s}, \tau\right), T_{1}^{2}\left(\tau, \tau_{s}\right)$ by $T_{1}^{1}, T_{1}^{2}$, respectively. We will use the same simplified notation for $n_{3}, n_{4}$ and $T_{j}^{i},(i=1,2, j=2, \cdots, 10)$ which will be introduced later.

When $\frac{\partial^{2} f_{i}\left(x_{1}^{*}, x_{2}^{*}\right)}{\partial x_{1}^{j} \partial x_{2}^{k}}=0$ and $\frac{\partial^{2} g_{i}\left(x_{1}^{*}, x_{2}^{*}\right)}{\partial x_{1}^{j} \partial x_{2}^{k}}=0$ for $j+k=2$, obviously $A_{20}=A_{11}=0$ and the flow on the centre manifold becomes

$$
\dot{\mathbf{z}}=B_{1} \mathbf{z}+\mathbf{\Psi}_{\mathbf{1}}(0) G_{3}\left(\boldsymbol{\Phi}_{\mathbf{1}} \mathbf{z}\right)+\text { h.o.t. }
$$

As discussed in section 2.3 (see eq. (20)) the normal form up to third order is

$$
\begin{aligned}
& \dot{w}_{1}=w_{2} \\
& \dot{w}_{2}=A_{30} w_{1}^{3}+A_{21} w_{1}^{2} w_{2}+\text { h.o.t. }
\end{aligned}
$$

Following a similar procedure to the quadratic case, we find that

$$
\begin{aligned}
& A_{30}=d_{1} F_{1}+d_{2} F_{2} \\
& A_{21}=d_{1} T_{2}^{1}+d_{2} T_{2}^{2}+3\left(d_{3} F_{1}+d_{4} F_{2}\right)
\end{aligned}
$$

where

$$
\begin{aligned}
F_{i}= & \sum_{j+k=3}\left(a_{i j k}+b_{i j k}\right) m_{1}^{k}, \\
T_{2}^{i}= & a_{i 21} m_{2}+2 a_{i 12} m_{1} m_{2}+3 a_{i 03} m_{1}^{2} m_{2}+3 b_{i 30} n_{1} \\
& +b_{i 21}\left(2 m_{1} n_{1}+n_{2}\right)+b_{i 12} m_{1}\left(m_{1} n_{1}+2 n_{2}\right)+3 b_{i 03} m_{1}^{2} n_{2} . \\
& \quad(i=1,2)
\end{aligned}
$$




\subsection{Triple zero Singularity}

From (7) a triple zero singularity can occur in (22) if (26), (27) and the following condition are satisfied:

$$
\begin{aligned}
\tau_{s}^{2}\left(a_{201} b_{110}+4 b_{110} b_{201}+a_{110} b_{201}\right)+2 \tau_{s}\left(b_{201}+b_{110}\right) & \\
-\tau^{2}\left(a_{210} b_{101}+4 b_{101} b_{210}+a_{101} b_{210}\right)+2 & =0,
\end{aligned}
$$

and $p^{\prime \prime \prime}(0) \neq 0$. Due to its length, we omit the expression for $p^{\prime \prime \prime}(0)$ here. In this case, the invariant subspace $P$ corresponding to the zero roots is three dimensional with basis $\Phi_{2}$ as given in (15). For convenience, we choose $\mathbf{v}_{1}, \mathbf{v}_{2}$ as for the BT singularity (see eq. (28)), and $\mathbf{v}_{3}=\left(\begin{array}{c}0 \\ m_{3}\end{array}\right)$ where

$$
m_{3}=-\frac{b_{110} \tau_{s}^{2}+b_{101} \tau^{2} m_{1}-b_{101} \tau m_{2}}{a_{101}+b_{101}} .
$$

The corresponding basis, $\Psi_{2}$, for the adjoint problem must have the form

$$
\Psi_{2}(s)=\left[\begin{array}{cc}
e_{1} \frac{s^{2}}{2}-e_{3} s+e_{5} & e_{2} \frac{s^{2}}{2}-e_{4} s+e_{6} \\
-e_{1} s+e_{3} & -e_{2} s+e_{4} \\
e_{1} & e_{2}
\end{array}\right]
$$

to satisfy $-\Psi_{2}^{\prime}=B_{2} \Psi_{2}$. The constants $e_{i}(i=1,2, \cdots, 6)$ can be determined by $<\Psi_{2}, \Phi_{2}>=$ I. This gives $\Psi_{2}(0)=\left[\begin{array}{ll}e_{5} & e_{6} \\ e_{3} & e_{4} \\ e_{1} & e_{2}\end{array}\right]$.

Applying the centre manifold projection to the DDE system (22) yields the three-dimensional ODE system

$$
\dot{\mathbf{z}}=B_{2} \mathbf{z}+\boldsymbol{\Psi}_{2}(0) G_{2}\left(\Phi_{2} \mathbf{z}\right)+\text { h.o.t. },
$$

where $\mathbf{z}=\left(\begin{array}{l}z_{1} \\ z_{2} \\ z_{3}\end{array}\right)$. As discussed in section 2.3 (see eq. (19)), the normal form of this equation is

$$
\begin{aligned}
& \dot{w}_{1}=w_{2}, \\
& \dot{w}_{2}=w_{3}, \\
& \dot{w}_{3}=A_{200} w_{1}^{2}+A_{110} w_{1} w_{2}+A_{101} w_{1} w_{3}+A_{020} w_{2}^{2}+\text { h.o.t. }
\end{aligned}
$$

To find the relationship between the coefficients of (38) and (39) we need to know the form of the near-identity transformation that relates them. This transformation is given in [38]. 
Using this transformation and $\mathbf{\Phi}_{\mathbf{1}} \mathbf{z}=\left(\begin{array}{c}z_{1}+\theta z_{2}+\frac{1}{2} \theta z_{3} \\ m_{1} z_{1}+\left(m_{2}+\theta m_{1}\right) z_{2}+\left(m_{3}+\theta m_{2}+\frac{1}{2} \theta m_{1}\right) z_{3}\end{array}\right)$, we find

$$
\begin{aligned}
& A_{200}=e_{1} E_{1}+e_{2} E_{2}, \\
& A_{110}=e_{1} T_{1}^{1}+e_{2} T_{1}^{2}+2\left(e_{3} E_{1}+e_{4} E_{2}\right), \\
& A_{101}=e_{1} T_{3}^{1}+e_{2} T_{3}^{2}+e_{3} T_{1}^{1}+e_{4} T_{1}^{2}+2\left(e_{5} E_{1}+e_{6} E_{2}\right), \\
& A_{020}=e_{1} T_{4}^{1}+e_{2} T_{4}^{2}+e_{3} T_{1}^{1}+e_{4} T_{1}^{2}+2\left(e_{5} E_{1}+e_{6} E_{2}\right),
\end{aligned}
$$

with $E_{i}, T_{1}^{i}(i=1,2)$ being given in $(33)$, and

$$
\begin{aligned}
T_{3}^{i}= & a_{i 11} m_{3}+2 a_{i 02} m_{1} m_{3}+2 b_{i 20} n_{3}+b_{i 11}\left(m_{1} n_{3}+n_{4}\right)+2 b_{i 02} m_{1} n_{4}, \\
T_{4}^{i}= & a_{i 02} m_{2}^{2}+b_{i 20} n_{1}^{2}+b_{i 11} n_{1} n_{2}+b_{i 02} n_{2}^{2}, \\
& \left(i=1,2, \quad n_{3}\left(\tau_{s}\right)=\frac{\tau_{s}^{2}}{2}, \quad n_{4}(\tau)=m_{3}-m_{2} \tau+m_{1} \frac{\tau^{2}}{2}\right) .
\end{aligned}
$$

Similar to the BT singularity, if $\frac{\partial^{2} f_{i}\left(x_{1}^{*}, x_{2}^{*}\right)}{\partial x_{1}^{j} \partial x_{2}^{k}}=0$ and $\frac{\partial^{2} g_{i}\left(x_{1}^{*}, x_{2}^{*}\right)}{\partial x_{1}^{j} \partial x_{2}^{k}}=0$ for $j+k=2$, then $A_{200}=A_{110}=A_{101}=A_{020}=0$. As discussed in section 2.3 (see eq. (21)) the normal form to third order is

$$
\begin{aligned}
& \dot{w}_{1}=w_{2} \\
& \dot{w}_{2}=w_{3} \\
& \dot{w}_{3}=A_{300} w_{1}^{3}+A_{210} w_{1}^{2} w_{2}+A_{120} w_{1} w_{2}^{2}+A_{030} w_{2}^{3}+A_{201} w_{1}^{2} w_{3}+A_{102} w_{1} w_{3}^{2}+\text { h.o.t. }
\end{aligned}
$$

Proceeding as in the quadratic case, we find

$$
\begin{aligned}
A_{300}= & e_{1} F_{1}+e_{2} F_{2}, \\
A_{210}= & e_{1} T_{2}^{1}+e_{2} T_{2}^{2}+3\left(e_{3} F_{1}+e_{4} F_{2}\right), \\
A_{120}= & e_{1} T_{5}^{1}+e_{2} T_{5}^{2}+2\left(e_{3} T_{2}^{1}+e_{4} T_{2}^{2}\right)+6\left(e_{5} F_{1}+e_{6} F_{2}\right), \\
A_{030}= & \frac{e_{1}}{3}\left(3 T_{6}^{1}-T_{7}^{1}\right)+\frac{e_{2}}{3}\left(3 T_{6}^{2}-T_{7}^{2}\right)+\frac{e_{3}}{3}\left(T_{5}^{1}-2 T_{8}^{1}\right)+\frac{e_{4}}{3}\left(T_{5}^{2}-2 T_{8}^{2}\right), \\
A_{201}= & e_{1} T_{8}^{1}+e_{2} T_{8}^{2}+e_{3} T_{2}^{1}+e_{4} T_{2}^{2}+3\left(e_{5} F_{1}+e_{6} F_{2}\right), \\
A_{102}= & \frac{e_{1}}{2}\left(2 T_{9}^{1}-T_{10}^{1}\right)+\frac{e_{2}}{2}\left(2 T_{9}^{2}-T_{10}^{2}\right)+\frac{e_{3}}{2}\left(T_{7}^{1}-3 T_{6}^{1}\right) \\
& +\frac{e_{4}}{2}\left(T_{7}^{2}-3 T_{6}^{2}\right)+\frac{e_{5}}{2}\left(2 T_{8}^{1}-T_{5}^{1}\right)+\frac{e_{6}}{2}\left(2 T_{8}^{2}-T_{5}^{2}\right),
\end{aligned}
$$


and

$$
\begin{aligned}
T_{5}^{i}= & a_{i 12} m_{2}^{2}+3 a_{i 03} m_{2}^{2} m_{1}+3 b_{i 30} n_{1}^{2} \\
& +b_{i 21} n_{1}\left(m_{1} n_{1}+2 n_{2}\right)+b_{i 12} n_{2}\left(2 m_{1} n_{1}+n_{2}\right)+3 b_{i 03} m_{1} n_{2}^{2}, \\
T_{6}^{i}= & a_{103} m_{2}^{3}+b_{i 30} n_{1}^{3}+b_{i 21} n_{1}^{2} n_{2}+b_{i 12} n_{1} n_{2}^{2}+b_{i 03} n_{2}^{3}, \\
T_{7}^{i}= & 2\left[a_{i 12} m_{2} m_{3}+3 a_{i 03} m_{1} m_{2} m_{3}\right. \\
& +3 b_{i 30} n_{1} n_{3}+b_{i 21}\left(m_{1} n_{1} n_{3}+n_{1} n_{4}+n_{2} n_{3}\right) \\
& \left.+b_{i 12}\left(m_{1} n_{2} n_{3}+m_{1} n_{1} n_{4}+n_{2} n_{4}\right)+3 b_{i 03} m_{1} n_{2} n_{4}\right], \\
T_{8}^{i}= & a_{i 21} m_{3}+2 a_{i 12} m_{1} m_{3}+3 a_{i 03} m_{1}^{2} m_{3} \\
& +3 b_{i 30} n_{3}+b_{i 21}\left(2 m_{1} n_{3}+n_{4}\right)+b_{i 12} m_{1}\left(m_{1} n_{3}+2 n_{4}\right)+3 b_{i 03} m_{1}^{2} n_{4}, \\
T_{9}^{i}= & a_{i 12} m_{3}^{2}+3 a_{i 03} m_{3}^{2} m_{1}+3 b_{i 30} n_{3}^{2} \\
& +b_{i 21} n_{3}\left(m_{1} n_{3}+2 n_{4}\right)+b_{i 12} n_{4}\left(2 m_{1} n_{3}+n_{4}\right)+3 b_{i 03} m_{1} n_{4}^{2}, \\
T_{10}^{i}= & 3 a_{i 03} m_{2}^{2} m_{3}+3 b_{i 30} n_{1}^{2} n_{3} \\
& +b_{i 21} n_{1}\left(n_{1} n_{4}+2 n_{2} n_{3}\right)+b_{i 12} n_{2}\left(n_{2} n_{3}+2 n_{1} n_{4}\right)+3 b_{030} n_{2}^{2} n_{4}, \\
& (i=1,2) .
\end{aligned}
$$

\section{Examples}

\subsection{Neural Network Model with Two Delays}

Here we apply our results to the neural network model considered in [30]. In that paper, the authors showed that Bogdanov-Takens points occurred in their model, but did not do the centre manifold analysis. The model (with a rescaling of the parameters) is given by the following system:

$$
\begin{aligned}
& \dot{x}(t)=-x(t)+a \tanh \left(x\left(t-\tau_{s}\right)\right)+a_{12} \tanh (y(t-\tau)), \\
& \dot{y}(t)=-y(t)+a_{21} \tanh (x(t-\tau))+a \tanh \left(y\left(t-\tau_{s}\right)\right) .
\end{aligned}
$$

Expanding Eq. (45), we have

$$
\begin{aligned}
& \dot{x}(t)=-x(t)+a x\left(t-\tau_{s}\right)+a_{12} y(t-\tau)-\frac{a}{3} x^{3}\left(t-\tau_{s}\right)-\frac{a_{12}}{3} y^{3}(t-\tau) \\
& \dot{y}(t)=-y(t)+a_{21} x(t-\tau)+a y\left(t-\tau_{s}\right)-\frac{a_{21}}{3} x^{3}(t-\tau)-\frac{a}{3} y^{3}\left(t-\tau_{s}\right)
\end{aligned}+\text { h.o.t. }
$$


The characteristic equation is

$$
p(\lambda)=(\lambda+1)^{2}-2(\lambda+1) a e^{-\tau_{s} \lambda}+a^{2} e^{-2 \tau_{s} \lambda}-a_{12} a_{21} e^{-2 \tau \lambda}=0 .
$$

When $a_{12} a_{21}=(a-1)^{2}$ and $\tau=\frac{1+a \tau_{s}}{a-1}$, then

$$
p(0)=p^{\prime}(0)=0,
$$

so the system has double zero (Bogdanov-Takens) singularity. As noted in [30], this will occur for $\tau>0$ if $a>1$ or $a<0$ and $\tau_{s}>-\frac{1}{a}$, and only the latter case occurs on the boundary of the region of stability of the trivial solution. Furthermore,

$$
p^{\prime \prime}(0)=-2\left(1+2 a \tau_{s}+a \tau_{s}^{2}\right) \neq 0 \text { if } a>1 \text { or } a<0, \tau_{s}>-\frac{1}{a},
$$

thus no triple zero singularity occurs.

Using the results of the previous section, the basis for the eigenspace corresponding to the double zero eigenvalue is, from (28),

$$
\Phi_{1}(\theta)=\left[\begin{array}{cc}
1 & \theta \\
\frac{1-a}{a_{12}} & \frac{1-a}{a_{12}} \theta
\end{array}\right],
$$

and the basis for the dual space, $\Psi_{1}(s)$, is given by (29) with

$$
\begin{array}{rlrl}
d_{1} & =\frac{a-1}{a \tau_{s}^{2}+2 a \tau_{s}+1}, & d_{2} & =-\frac{a_{12}}{a \tau_{s}^{2}+2 a \tau_{s}+1}, \\
d_{3}=\frac{\left(\left(a \tau_{s}+1\right)^{3}-a(a-1)^{2} \tau_{s}^{3}\right.}{3\left(a \tau_{s}^{2}+2 a \tau_{s}+1\right)^{2}} & d_{4}= & -\frac{a_{12}\left(\left(a \tau_{s}+1\right)^{3}-a(a-1)^{2} \tau_{s}^{3}\right.}{3(a-1)\left(a \tau_{s}^{2}+2 a \tau_{s}+1\right)^{2}} .
\end{array}
$$

Since the nonlinear functions are odd functions, we compute the normal form up to third order:

$$
\begin{aligned}
& \dot{w}_{1}=w_{2}, \\
& \dot{w}_{2}=A_{30} w_{1}^{3}+A_{21} w_{1}^{2} w_{2},
\end{aligned}
$$

where

$$
A_{30}=-\frac{(a-1)\left(a_{12}^{2}+(a-1)^{2}\right)}{3 a_{12}^{2}\left(a \tau_{s}^{2}+2 a \tau_{s}+1\right)}, A_{21}=-\frac{\left(a_{12}^{2}+(a-1)^{2}\right)\left(a(2 a-1)\left(\tau_{s}+1\right)^{3}-2(a-1)^{2}\right)}{3 a_{12}^{2}\left(a \tau_{s}^{2}+2 a \tau_{s}+1\right)^{2}} .
$$

Noting that $a \tau_{s}^{2}+2 a \tau_{s}+1=\left(a \tau_{s}+1\right)\left(\tau_{s}+1\right)+(a-1) \tau_{s}$, we see that $A_{30}<0$ when $a<0$ and $\tau_{s}>-\frac{1}{a}$. Further since $\tau_{s}>-\frac{1}{a}$, we have

$$
\begin{aligned}
a(2 a-1)\left(\tau_{s}+1\right)^{3} & >a(2 a-1)(1-1 / a)^{3} \\
& =2 a^{2}-7 a+9-\frac{5}{a}+\frac{1}{a^{2}} \\
& =2(a-1)^{2}-3 a+7-\frac{5}{a}+\frac{1}{a^{2}} .
\end{aligned}
$$


Thus $A_{21}<0$ as well.

The unfolding of the normal form (46) is

$$
\begin{aligned}
& \dot{w}_{1}=w_{2}, \\
& \dot{w}_{2}=\mu_{1} w_{1}+\mu_{2} w_{2}+A_{30} w_{1}^{3}+A_{21} w_{1}^{2} w_{2} .
\end{aligned}
$$

This system is well understood. The complete set of bifurcations for any values of $A_{30}$ and $A_{21}$ is given in the book of Guckenheimer and Holmes [14, Section 7.3]. In particular, they show that when $A_{30}<0$ and $A_{21}<0$, there is a region in the $\mu_{1}, \mu_{2}$ parameter plane where a pair of stable equilibria coexist with a large amplitude stable limit cycle. The period of the limit cycle may be large as the region of bistability ends near a homoclinic bifurcation. This region of bistability does not occur for other signs of the normal form coefficients. Bistability between a pair of equilibrium points and a slowly oscillating limit cycle was observed by Shayer and Campbell (cf. [30, Fig. 8]) in numerical simulations of the full nonlinear DDE (45) with the parameter values $a=-2, \tau_{s}=0.75, \tau=0.1, a_{12}=2 a_{21}=4.68$. Engelborghs et al. used the numerical bifurcation for package they developed, DDE-BIFTOOL [7], to study the homoclinic bifurcation of periodic orbits in this model with similar parameter values $[6]$.

To further support our analysis, we have performed numerical continuation studies of the full system (45) using DDE-BIFTOOL. These results are illustrated in Figures 1-2. We find three secondary bifurcations emanating from the Bogdanov-Takens point. As $a_{21}$ is increased with $\tau$ less that the value at the BT point these bifurcations are as follows. A Hopf bifurcation of the nontrivial equilibria (denoted $\mathrm{H}_{\mathrm{NT}}$ in the figures) creates a pair of limit cycles surrounding the nontrivial equilibria. These limit cycles then undergo a bifurcation (HL) in which they form a double homoclinic loop to the origin and then become a single large limit cycle surrounding both nontrivial equilibria. Finally, this large limit cycle is destroyed in a saddle node bifurcation of limit cycles (SNLC) with the limit cycle from the primary Hopf bifurcation $\left(\mathrm{H}_{0}\right)$. This sequence of bifurcations is exactly that presented in [14, Section 7.3]. A schematic of the sequence of phase portraits associated with these bifurcations can be seen in [14, Figure 7.3.9]. 


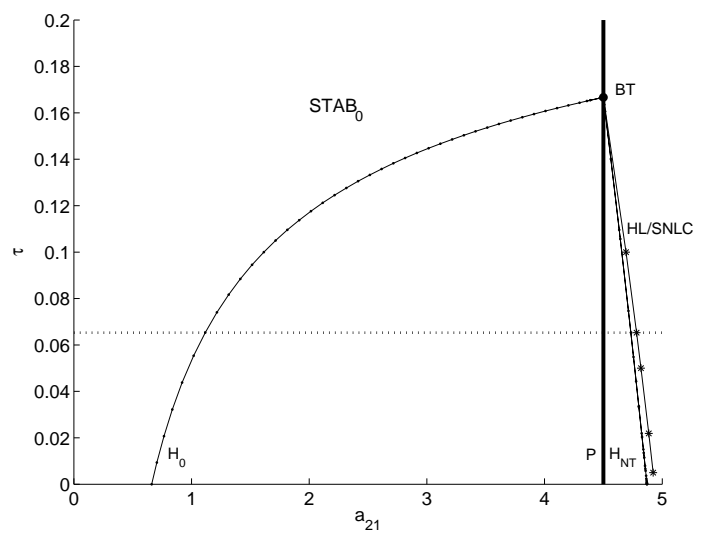

Figure 1: Bifurcation curves of equation (45) in $a_{21}, \tau$ parameter space. Other parameter values are: $a=-2, a_{12}=2, \tau_{s}=0.75$. $\mathrm{P}$ - pitchfork bifurcation, $\mathrm{H}_{0} / \mathrm{H}_{\mathrm{NT}}$ - Hopf bifurcation of trivial/nontrivial equilibrium, HL - homoclinic loop bifurcation of periodic orbits surrounding nontrivial equilibria, SNLC saddle node of limit cycles bifurcation, BT - Bogdanov-Takens point. The stability region of the trivial solution is denoted by $\mathrm{STAB}_{0}$. The HL and SNLC curves are too close together to resolve at this scale.

\subsection{Fitzhugh/Nagumo Equation with One Delay}

Plant [27] considered a model for a recurrent neural circuit which consisted of a single Fitzhugh Nagumo neuron with delayed feedback on the voltage. We will study a similar model here and show that it exhibits both the double zero and triple zero singularity. Our neural model is a slight modification of the Fitzhugh Nagumo model due to Murray [24]. Including the delayed feedback leads to the following system of equations:

$$
\begin{aligned}
\dot{v} & =v(t)(a-v(t))(v(t)-1)-w(t)+\mu v\left(t-\tau_{s}\right) \\
\dot{w} & =b v(t)-\gamma w(t)
\end{aligned}
$$

The parameters are restricted to $0<a<1$ and $\tau_{s} \geq 0$, for physical reasons.

Clearly, $(0,0)$ is an equilibrium point of the system for all parameter values. The characteristic equation of the linearization of (48) about this trivial equilibrium point is

$$
P(\lambda)=\lambda^{2}+(\gamma+a) \lambda+a \gamma-\mu e^{-\tau_{s} \lambda} \lambda-\mu e^{-\tau_{s} \lambda} \gamma+b=0
$$

It is easy to see that when the parameters satisfy

$$
\mu=a+\frac{b}{\gamma} \stackrel{\text { def }}{=} \mu_{*},
$$




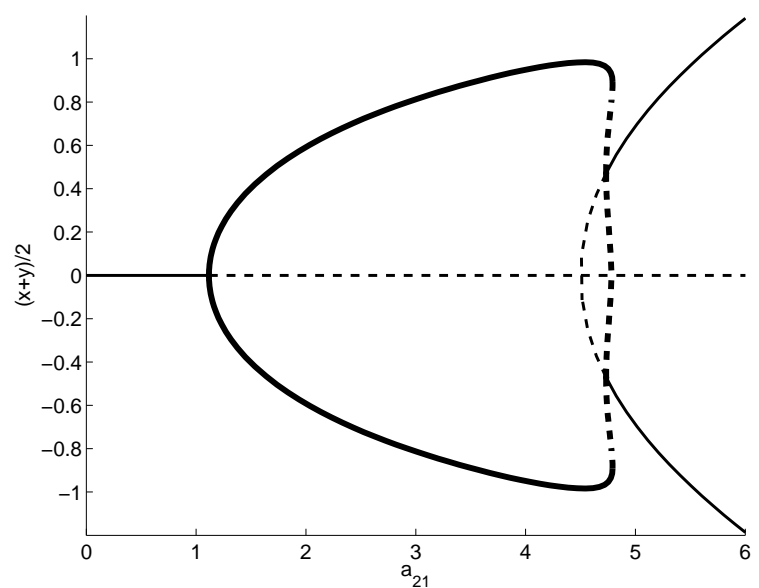

(a)

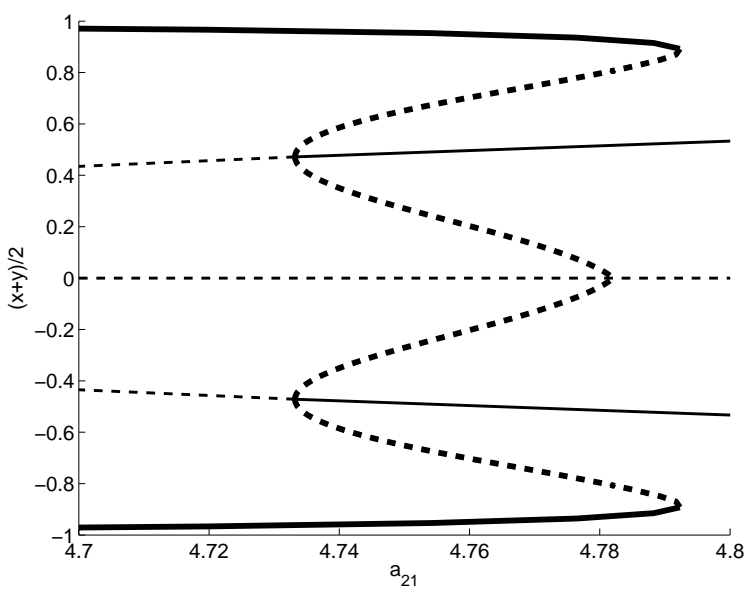

(b)

Figure 2: Bifurcations of equation (45) as $a_{21}$ is varied with $\tau=0.0653, a=-2, a_{12}=$ $2, \tau_{s}=0.75$. This corresponds to dotted line in Figure 1. Thin lines represent equilibria, thick lines periodic orbits. Solid/dashed lines represents stable/unstable objects. Periodic orbits are represented by their maximum and minimum values. (a) Full diagram. (b) Zoom in illustrating the three secondary bifurcations. Labels are as for Figure 1.

the characteristic equation has a zero root. A check of the standard conditions [26] shows that this corresponds to a transcritical bifurcation of the trivial solution. If the parameters also satisfy

$$
\tau_{s}=\frac{b-\gamma^{2}}{\gamma(a \gamma+b)} \stackrel{\text { def }}{=} \tau_{*}
$$

and $b^{2}+2 \gamma(\gamma+a) b-\gamma^{4} \neq 0$, there exists double zero singularity in the system (48). Finally, if the parameters satisfy (49), (50),

$$
b=-\gamma(a+\gamma) \pm \gamma \sqrt{(a+\gamma)^{2}+\gamma^{2}} \stackrel{\text { def }}{=} b_{* \pm},
$$

$\gamma \neq-a$ and $\gamma \neq-\frac{3 a}{7}$, there exists a triple zero singularity in (48).

For the double zero singularity to occur in the physically reasonable part of parameter space, we require $\tau_{*} \geq 0$. This will occur under the following constraints of the parameters

1. $\gamma>0$ and $b>\gamma^{2}$

2. $\gamma>0$ and $b<-a \gamma<0$

3. $-a<\gamma<0$ and $\gamma^{2}<b<-a \gamma$ 
4. $\gamma<-a$ and $-a \gamma<b<\gamma^{2}$.

By considering the stability of the equilibrium with $\tau_{s}=0$, one can show that case 3 does not lie on the boundary of the stability region of the equilibrium point. Hence the double zero point in this case will not affect the observable dynamics of the system. Further, it can be shown that in cases 1 and 4 the triple zero singularity does not occur. Thus we will concentrate on case 2 from now on. Noting that the triple zero singularity occurs at $b=b_{*-}$ in this case, we will denote $b_{*-}=b_{*}$.

For double zero singularity, we choose the basis in the two-dimensional subspace $P$ as

$$
\Phi(\theta)=\left[\begin{array}{cc}
1 & \theta \\
\frac{b}{\gamma} & -\frac{b}{\gamma^{2}}+\frac{b}{\gamma} \theta
\end{array}\right],
$$

then the coefficients in the corresponding basis

$$
\Psi(s)=\left[\begin{array}{cc}
-l_{1} s+l_{3} & -l_{2} s+l_{4} \\
l_{1} & l_{2}
\end{array}\right]
$$

are

$$
\begin{array}{rlrl}
l_{1} & =-\frac{2 \gamma}{\gamma \mu_{*} \tau_{*}^{2}-2 \mu_{*} \tau_{*}-2}, & l_{2} & =\frac{2 \gamma^{2}\left(\mu_{*} \tau_{*}+1\right)}{b\left(\gamma \mu_{*} \tau_{*}^{2}-2 \mu_{*} \tau_{*}-2\right)}, \\
l_{3}=-\frac{2\left[\gamma^{2} \mu_{*} \tau_{*}^{3}-6\left(\mu_{*} \tau_{*}+1\right)\right]}{3\left(\gamma \mu_{*} \tau_{*}^{2}-2 \mu_{*} \tau_{*}-2\right)^{2}}, & l_{4}=\frac{2 \gamma^{2} \mu_{*} \tau_{*}^{2}\left(\mu_{*} \tau_{*}+1\right)\left(\gamma \tau_{*}-3\right)}{3 b\left(\gamma \mu_{*} \tau_{*}^{2}-2 \mu_{*} \tau_{*}-2\right)^{2}} .
\end{array}
$$

We can obtain the second-order normal form as

$$
\begin{aligned}
& \dot{w}_{1}=w_{2} \\
& \dot{w}_{2}=l_{1}(a+1) w_{1}^{2}+2 l_{3}(a+1) w_{1} w_{2} .
\end{aligned}
$$

The unfolding consistent with the transcritical bifurcation in the orginal system, (48), is (see $[14$, Section 7.3])

$$
\begin{aligned}
& \dot{w}_{1}=w_{2} \\
& \dot{w}_{2}=\mu_{1} w_{1}+\mu_{2} w_{2}+l_{1}(a+1) w_{1}^{2}+2 l_{3}(a+1) w_{1} w_{2} .
\end{aligned}
$$

The behaviour of this unfolding is similar to the more common case where the steady state bifurcation associated with the double zero singularity is a saddle node bifurcation. For simplicity in our description of this behaviour, we define

$$
\alpha=l_{1}(a+1), \beta=2 l_{3}(a+1)
$$


and note that since $a>0$, the signs of these quantities are determined by $l_{1}$ and $l_{3}$, respectively. Analysis similar to that found in, for example, [14, Section 7.3] shows that there are five bifurcation curves emanating from the origin in the $\mu_{1}, \mu_{2}$ parameter space:

- a transcritical bifurcation at $\mu_{1}=0$,

- a Hopf bifurcation of the trivial equilibrium at $\mu_{2}=0, \mu_{1}<0$,

- a Hopf bifurcation of the the nontrivial equilibrium along a curve which is tangent at the origin to $\mu_{2}=\frac{\beta}{\alpha} \mu_{1}, \mu_{1}>0$,

- a homoclinic bifurcation leading to the creation of a periodic orbit about the trivial solution along a curve which is tangent at the origin to $\mu_{2}=\frac{\beta}{7 \alpha} \mu_{1}, \mu_{1}<0$,

- a homoclinic bifurcation leading to the creation of a periodic orbit about the nontrivial equilibrium point along a curve which is tangent at the origin to $\mu_{2}=\frac{6 \beta}{7 \alpha} \mu_{1}, \mu_{1}>0$.

The signs of $l_{1}$ and $l_{3}$ determine where these bifurcations occur, the criticality of the Hopf bifurcations and the stability of the periodic orbits created by the homoclinic orbits. In particular, when $l_{1} l_{3}>0$ both Hopf bifurcations are subcritical and when $l_{1} l_{3}<0$ they are both supercritical. There are only four possible unfoldings, two of which are pictured in Figure 3. The others may be obtained from these by noting that the equations are unchanged by the transformation $l_{j} \rightarrow-l_{j}, w_{j} \rightarrow-w_{j}$.

Using the expressions for $\mu_{*}$ and $\tau_{*}$ we can rewrite those for $l_{1}, l_{3}$ as

$$
l_{1}=\frac{2 \gamma^{3}(b+a \gamma)}{b^{2}+2(a+\gamma) b \gamma-\gamma^{4}}, \quad l_{3}=\frac{2 \gamma^{2}\left(5 b^{3}+3(4 a+\gamma) b^{2} \gamma+3\left(2 a^{2}-\gamma^{2}\right) b \gamma^{2}+\gamma^{6}\right)}{3\left(b^{2}+2(a+\gamma) b \gamma-\gamma^{4}\right)^{2}}
$$

Note that both $l_{1}$ and $l_{3}$ have zero denominator at $b=b_{*}$ and that $l_{1}$ changes sign at $b_{*}$ but $l_{3}$ does not. Further, $b_{*}<-a \gamma$, and hence $l_{1}<0$ for $b<b_{*}$ and $l_{1}>0$ for $b_{*}<b<-a \gamma$. Recall that for $b>-a \gamma$ we have $\tau_{*}<0$. Since the numerator of $l_{3}$ is a cubic in $b$ it will change sign at least once as $b$ is varied.

Taking the specific parameter values $a=0.2, \gamma=1$, we find that $l_{3}$ changes sign once at $b=\hat{b} \approx-1.527$. The sign of the coefficients and the corresponding unfoldings are given in the following table. 


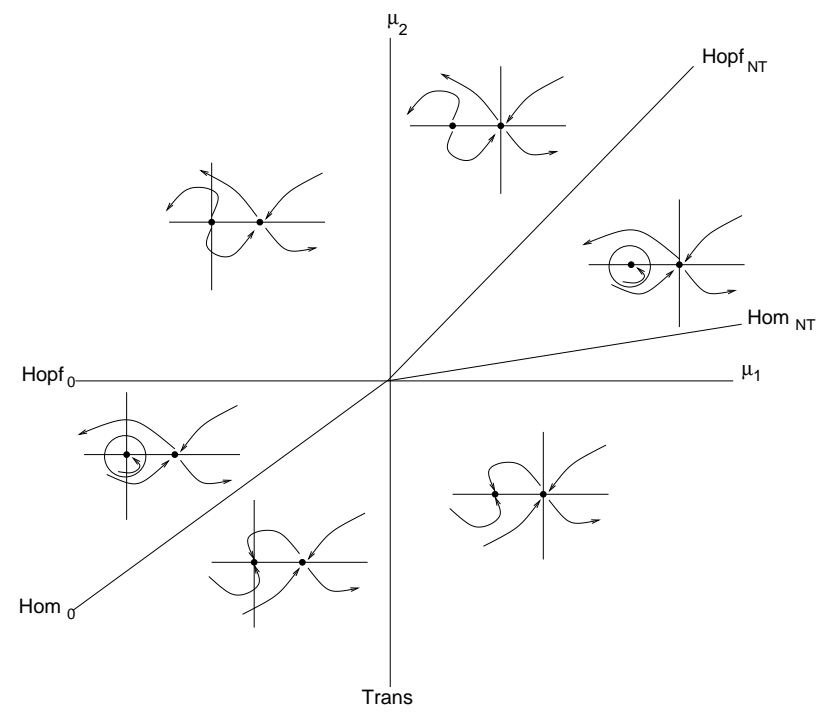

(a) $l_{1}>0, l_{3}>0$

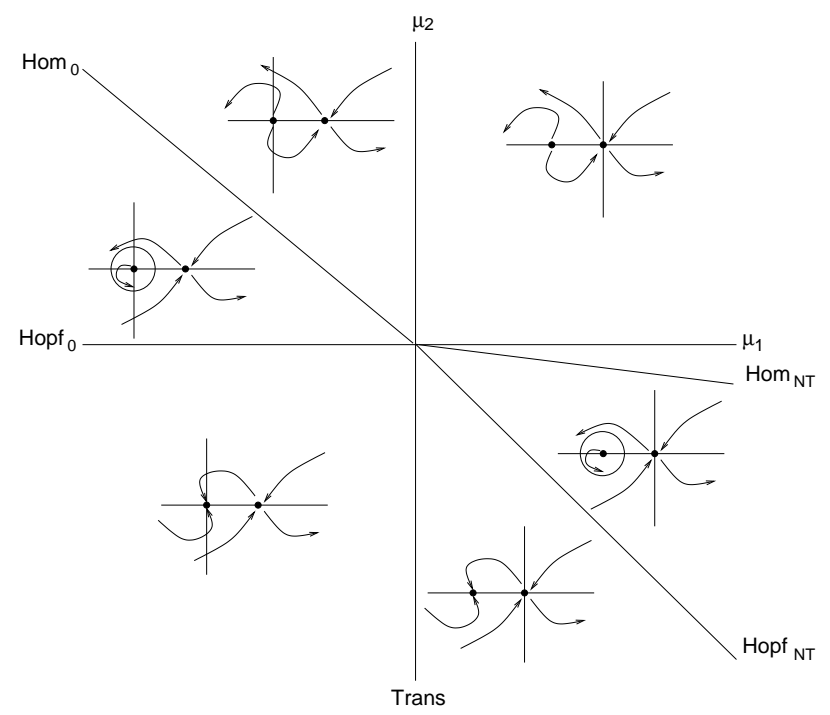

(b) $l_{1}>0, l_{3}<0$

Figure 3: Possible unfoldings of the double zero with a transcritical bifurcation. Trans transcritical bifurcation, Hom- homoclinic bifurcation. Subscripts refer to equilibrium type: 0 - trivial, NT - nontrivial.

\begin{tabular}{|c|c|c|}
\hline Range in $b$ & Signs of coefficients & Unfolding \\
\hline$b<b_{*} \approx-2.76$ & $l_{1}<0, l_{3}<0$ & Figure 3(a), with $w_{j} \rightarrow-w_{j}$ \\
$b_{*}<b<\hat{b} \approx-1.527$ & $l_{1}>0, l_{3}<0$ & Figure 3(b) \\
$\hat{b}<b<-a \gamma=-0.2$ & $l_{1}>0, l_{3}>0$ & Figure 3(a) \\
\hline
\end{tabular}

Numerical continuations (using DDE-BIFTOOL [7]) of the full equation (48) confirm the predictions of the normal form analysis. Figure 4 shows three continuations of the periodic orbits created from the Hopf bifurcation of the trivial solution. For $b=-3.1$ and $b=-1$ the Hopf bifurcation is subcritical, for $b=-2.5$ it is supercritical. All of the periodic orbits are eventually lost in homoclinic bifurcations as predicted by the normal form. Note that for $b=-3.1$ the equilibrium point and periodic orbit are unstable before and after the bifurcation as the Hopf bifurcation point does not lie on boundary of the region of stability. Figure 5 shows a two parameter bifurcation diagram in the case $b=-2.5$. We have confirmed the presence of three secondary bifurcations emanating from the Bogdanov-Takens point: a homoclinic bifurcation of the periodic orbit surrounding the trivial equilibrium point $\left(\operatorname{Hom}_{0}\right)$, a Hopf bifurcation of the nontrivial equilibrium point $\left(\operatorname{Hopf}_{\mathrm{NT}}\right)$ and a homoclinic bifurcation of the periodic orbit surrounding the nontrivial equilibrium point $\left(\operatorname{Hom}_{\mathrm{NT}}\right)$. Note the good 

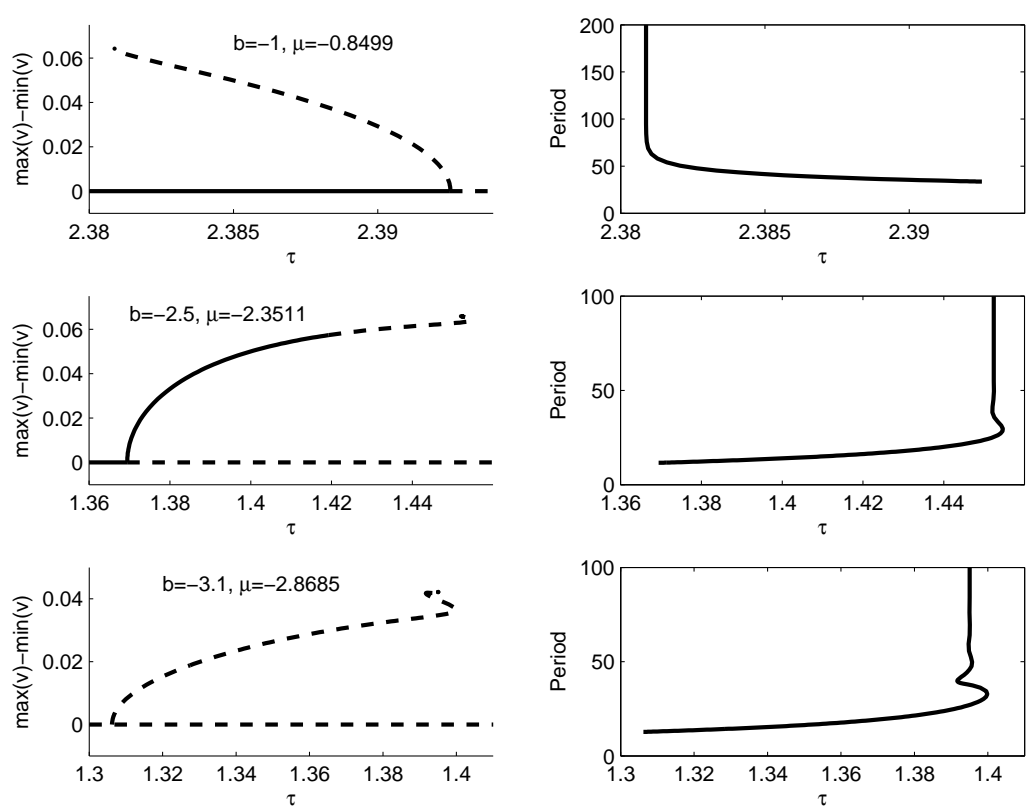

Figure 4: Numerical bifurcation diagrams showing bifurcations as $\tau$ is varied. In each diagram a periodic solution is created via a Hopf bifurcation from the trivial solution and subsequently destroyed in a homoclinic bifurcation. Parameter values are $a=0.2, \gamma=1$ and $b, \mu$ as shown. Solid lines indicate stable equilibria/periodic orbits and dashed lines indicate unstable ones. Left: amplitude of solutions. Right: period of solutions.

correspondence with the prediction from normal form theory shown in Figure 3(b).

For triple zero singularity, we can select the basis in the three-dimensional subspace $P$ as

$$
\Phi(\theta)=\left[\begin{array}{ccc}
1 & \theta & \frac{\theta^{2}}{2} \\
\frac{b_{*}}{\gamma} & -\frac{b_{*}}{\gamma^{2}}+\frac{b_{*}}{\gamma} \theta & \frac{\left(b_{*}-\gamma^{2}\right)^{2}}{\gamma^{3}\left(a \gamma+b_{*}\right)}+\frac{-b_{*}}{\gamma^{2}} \theta+\frac{b_{*}}{\gamma} \frac{\theta^{2}}{2}
\end{array}\right],
$$

then the basis in the associated dual subspace is

$$
\Psi(s)=\left[\begin{array}{cc}
e_{1} \frac{s^{2}}{2}-e_{3} s+e_{5} & e_{2} \frac{s^{2}}{2}-e_{4} s+e_{6} \\
-e_{1} s+e_{3} & -e_{2} s+e_{4} \\
e_{1} & e_{2}
\end{array}\right]
$$

where

$$
\begin{aligned}
e_{1} & =-\frac{6\left(\mu_{*}-a\right)}{\tau_{*}^{2} \mu_{*}\left(5 \tau_{*} \mu_{*}+\tau_{*} a+6\right)}, \\
e_{3} & =-\frac{12\left[109 \tau_{*}^{2} \mu_{*}^{3}+\left(10 \tau_{*} a+51\right) \tau_{*} \mu_{*}^{2}+\left(\tau_{*}^{2} a^{2}+9 \tau_{*} a-57\right) \mu_{*}-3 a\right]}{5 \tau_{*} \mu_{*}\left(5 \tau_{*} \mu_{*}+\tau_{*} a+6\right)\left[7 \tau_{*}^{2} \mu_{*}^{2}-\left(\tau_{*}+24\right) \tau_{*} \mu_{*}+6\right]},
\end{aligned}
$$




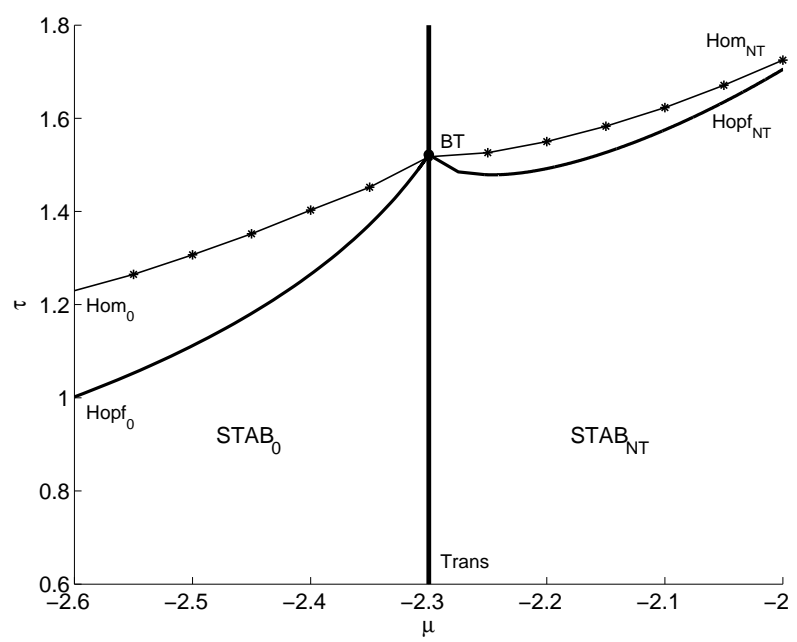

Figure 5: Bifurcations curves in $\mu, \tau$ parameter space generated by numerical continuation of system (48) with $\gamma=1, a=0.2$ and $b=-2.5$. The picture would be qualitatively similar for any $b \in\left(b^{*}, \hat{b}\right) \approx(-2.76,-1.527)$. STAB denotes stability region of trivial, 0 , or nontrivial, NT, equilibrium. Other labels are as for Figure 3.

$$
\begin{aligned}
e_{5} & =-\frac{3\left[313 \tau_{*}^{2} \mu_{*}^{2}+2\left(23 a \tau_{*}+126\right) \tau_{*} \mu_{*}+\tau_{*}^{2} a^{2}-12 \tau_{*} a-120\right]}{10\left[35 \tau_{*}^{3} \mu_{*}^{3}+2\left(a \tau_{*}-39\right) \tau_{*}^{2} \mu_{*}^{2}-\left(a^{2} \tau_{*}^{2}+30 a \tau_{*}+114\right) \tau_{*} \mu_{*}+6 a \tau_{*}+36\right]} \\
& \vdots
\end{aligned}
$$

We omit the expressions for the other coefficients $e_{i}, i=2,4,6$ since they do not appear in the coefficients of the normal form.

The second-order normal form for the triple zero singularity is Eq. (38) with the coefficients are

$$
\begin{array}{ll}
A_{200}=(a+1) e_{1}, & A_{110}=2(a+1)\left(e_{1}+e_{3}\right), \\
A_{101}=2(a+1)\left(e_{1}+e_{3}+e_{5}\right), & A_{020}=(a+1)\left(e_{1}+2 e_{3}+2 e_{5}\right) .
\end{array}
$$

The unfolding of this is (see [31])

$$
\begin{aligned}
& \dot{w}_{1}=w_{2}, \\
& \dot{w}_{2}=w_{3}, \\
& \dot{w}_{3}=\mu_{1} w_{1}+\mu_{2} w_{2}+\mu_{3} w_{3}+A_{200} w_{1}^{2}+A_{110} w_{1} w_{2}+A_{101} w_{1} w_{3}+A_{020} w_{2}^{2} .
\end{aligned}
$$

We have the following results for the unfolding normal form (52). 
- The origin is stable when $\mu_{i}<0,(i=1,2,3)$ and $\mu_{1}>-\mu_{2} \mu_{3}$;

- The origin undergoes a Hopf bifurcation when $\mu_{3}=-\frac{\mu_{1}}{\mu_{2}}$ and $\mu_{2}<0$;

- The origin undergoes a transcritical bifurcation at $\mu_{1}=0$;

- For $\mu_{1} \neq 0$, there is a nontrivial equilibrium point at $\mathbf{z}^{*}=\left(-\mu_{1} / A_{200}, 0,0\right)$;

- The nontrivial equilibrium point, $\mathbf{z}^{*}$, undergoes a Hopf bifurcation when $\mu_{3}=\mu_{1}\left(A_{101} / A_{200}-A_{200} /\left(A_{110} \mu_{1}-A_{200} \mu_{2}\right)\right)$ and $A_{200} /\left(A_{110} \mu_{1}-A_{200} \mu_{2}\right)>0 ;$

- The origin undergoes a BT bifurcation when $\mu_{1}=\mu_{2}=0$;

- The origin undergoes a Transcritical/Hopf bifurcation when $\mu_{1}=\mu_{3}=0$ and $\mu_{2}<0$.

There will also be two surfaces of Homoclinic bifurcation: one creating a periodic orbit about the trivial solution and one creating a periodic orbit about the nontrivial solution. To see this, note that on each plane $\mu_{3}=K \neq 0$ the origin is a BT point, in which case system (52) may be reduced to system (51).

For system (48) the surface of transcritical bifurcation is given by equations (49). The equation for the surface of Hopf bifurcation of trivial solution is given by the following equations, defined parametrically in terms of the Hopf bifurcation frequency $\omega$,

$$
\mu=\frac{\sqrt{\mathcal{S}(\omega)^{2}+\mathcal{C}(\omega)^{2}}}{\gamma^{2}+\omega^{2}}, \quad \tau_{s}=\frac{1}{\omega} \arctan \left(\frac{\mathcal{S}(\omega)}{\mathcal{C}(\omega)}\right)
$$

where

$$
\mathcal{S}(\omega)=\omega\left(b-\left(\omega^{2}+\gamma^{2}\right)\right) \text { and } \mathcal{C}(\omega)=\gamma(a \gamma+b)+\omega^{2} a
$$

These surfaces are illustrated in Figure 6 for $a=0.2, \gamma=1$, with the light grey surface corresponding to the transcritical bifurcation and the dark grey surface to the Hopf bifurcation. The codimension two points occur along the curves of intersection of these surfaces. The curve of double zero singularities is marked by a solid line and the curve of zero/pure imaginary pair singularities is marked by a dashed line. The intersection point of these two curves is the triple zero singularity point. 


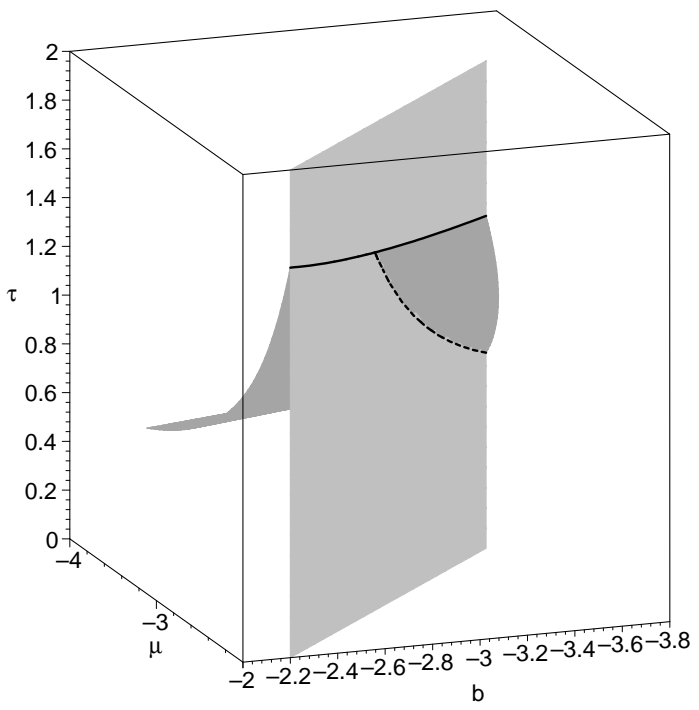

Figure 6: Bifurcation surfaces in $\mu, \tau, b$ parameter space for system (48) with $\gamma=1, a=0.2$. Shown are: surfaces of Hopf bifurcation (dark grey) and transcritical bifurcation (light grey), curves of double zero singularities (solid) and zero/pure imaginary pair singularities (dashed). The triple zero singularity occurs at the intersection of these latter two curves.

\section{Conclusions}

We have studied the double zero (Bogdanov-Takens) and triple zero singularities with geometric multiplicity one in a general system of two delay differential equations with two time delays. Our results are given in terms of the coefficients of the Taylor series of the right hand side of the general DDE about an equilibrium point, and thus can be easily applied to any model which can be written in this form. In particular, we have given conditions for the existence of each type of singularities. We have also given formulas for the coefficients of the normal form for each singularity, both in the generic case and the case where the quadratic terms of the Taylor series are zero. This latter case occurs, for example, when the nonlinearities in the model are odd functions.

Our results apply to a large variety of nonlinear dynamical models. We have given detailed studies of the application of the results to two of these models: an artificial neural 
network model [30] and a model for a delayed recurrent neural circuit similar to that studied by $[27]$.

The neural network model can exhibit the double zero singularity but not the triple zero singularity. We have given constraints on the parameters so that double zero singularity occurs on the boundary of the region of stability of the equilibrium point and showed that under these constraints, both coefficients of the normal form must negative. We confirmed, via numerical bifurcation analysis, the presence of all the secondary bifurcations predicted by the normal form theory. The main implication of the double zero singularity for the dynamics of the system is the existence of a region in parameter space where bistability between a pair of nontrivial equilibrium points and a large amplitude (and possibly slowly oscillating) periodic solution. This bistability means that the neural network can exhibit oscillator death, i.e. a correctly applied perturbation may cause the system to stop oscillating and settle on an equilibrium point.

The recurrent neural circuit model is more interesting for two reasons. First, it can exhibit both the double and triple zero singularities. Second, due to the form of the model, the steady state bifurcation associated with these singularities is a transcritical bifurcation, instead of the usual (generic) saddle node bifurcation. We determined and analyzed the unfoldings of the normal forms (with arbitrary coefficients) for both singularities. The secondary bifurcations resulting from the double zero singularity are similar to those in the case of a saddle node bifurcation, however, no region of bistability occurs. The unfolding of the triple zero singularity reveals that surfaces of Hopf bifurcation and transcritical bifurcation must emanate from the singularity point. Further, two curves of codimension two bifurcations occur: Bogdanov-Takens bifurcations (corresponding to the double zero singularity) and Transcritical/Hopf bifurcations. We also argue that two surfaces of homoclinic bifurcations must occur. Analysis of the model shows that the Hopf, Transcritical and the codimension two bifurcations occur as predicted by the normal form. The triple zero singularity acts as an organizing centre for the dynamics of the model. 


\section{References}

[1] A. Algaba, E. Freire, And E. Gamero, Characterizing and computing normal forms using Lie transforms: a survey., Dyn. Contin. Discrete Impuls. Syst. Ser. A, 8 (2001), pp. 449-475.

[2] R. Bogdanov, Versal deformations of a singular point on the plane in the case of zero eigenvalues, Func. Anal. Appl., 9 (1975), pp. 144-145.

[3] R. Cushman And J. SAnders, Nilpotent normal forms and representation theory of $s l(2, r)$, in Multiparameter Bifurcation Theory, M. Golubitsky and J. Guckenheimer, eds., vol. 56 of Contemporary Mathematics, AMS, 1986, pp. 31-51.

[4] F. Dumortier And S. IBÁÑEz, Nilpotent singularities in generic 4-parameter families of 3-dimensional vector fields, J. Diff. Eqs., 127 (1996), pp. 590-647.

[5] F. Dumortier, S. IbÁÑez, And H. Kokubu, New aspects in the unfolding of the nilpotent singularity of codimension three, Dyn. Sys., 16 (2001), pp. 63-95.

[6] K. Engelborghs, T. Luzyanina, And D. Roose, Numerical bifurcation analysis of delay differential equations using DDE-BIFTOOL, ACM Transactions on Mathematical Software, 28 (2002), pp. 1-21.

[7] K. Engelborghs, T. Luzyanina, and G. Samaey, DDE-BIFTOOL v. 2.00: $a$ matlab package for bifurcation analysis of delay differential equations., Tech. Rep. TW330, Department of Computer Science, K.U. Leuven, Leuven, Belgium, 2001.

[8] H. ERZGRÄBER ET AL., Frequency versus relaxation oscillators in a semiconductor laser with coherent filtered optical feedback, Phys. Rev. E, 73 (2006).

[9] T. FARIA, On a planar system modeling a neuron network with memory, J. Diff. Eqs., 168 (2000), pp. 129-149.

[10] — On the study of singularities for a plane system with two delays, J. Dyn. Contin. Discrete Impuls. Syst., Ser. A, 10 (2003), pp. 357-371. 
[11] T. Faria And L. Magalhães, Normal forms for retarded functional differential equations and applications to Bogdanov-Takens singularity, J. Diff. Eqs., 122 (1995), pp. 201224.

[12] E. Freire, E. Gamero, and A. Rodriguez-Luis, A note on the triple-zero linear degeneracy: normal forms, dynamical and bifurcation behaviors of an unfolding, Internat. J. Bifur. Chaos, 12 (2002), pp. 2799-2820.

[13] F. Giannakopoulos And A. Zapp, Bifurcations in a planar system of differential delay equations modeling neural activity, Physica D, 159 (2001), pp. 215-232.

[14] J. Guckenheimer And P. Holmes, Nonlinear Oscillations, Dynamical Systems and Bifurcations of Vector Fields, Springer-Verlag, New York, 1983.

[15] S. Guo And L. Huang, Hopf bifurcating periodic orbits in a ring of neurons with delays, Phys. D, 183 (2003), pp. 19-44.

[16] J. HALE, Flows on center manifolds for scalar functional differential equations, Proc. R. Soc. Edinburgh, 101A (1985), pp. 193-201.

[17] J. Hale and S. Verduyn Lunel, Introduction to Functional Differential Equations, Springer Verlag, New York, 1993.

[18] S. IbÁÑEz AND J. RODRIGUEZ, Sil'nikov bifurcations in generic 4-unfoldings of codimension-4 singularity, J. Diff. Eqs., 120 (1995), pp. 411-428.

[19] _ Sil'nikov configurations in any generic unfolding of the nilpotent singularity of codimension three on $R^{3}$, J. Diff. Eqs., 208 (2005), pp. 147-175.

[20] G. Iooss And M. Adelmeyer, Topics in Bifurcation Theory and Applications, World Scientific, Singapore, 1992.

[21] Y. Kuznetsov, Elements of Applied Bifurcation Theory, vol. 112 of Applied Mathematical Sciences, Springer-Verlag, Berlin/New York, 1995. 
[22] Z. LiU AND R. YUAN, Bifurcations in predator-prey system with nonmonotonic functional response, Nonlinear Analysis: Real World Applications, 6 (2005), pp. 187-205.

[23] M. Medved, On a codimension three bifurcation, Casopis Pro Pestovani Matematiky, 109 (1984), pp. 3-26.

[24] J. Murray, Mathematical Biology, Springer-Verlag, New York, 1989.

[25] G. Orosz And G. StÉPÁn, Subcritical hopf bifurcations in a car-following model with reaction-time delay, Proceedings of the Royal Society of London A, 462 (2006).

[26] L. Perko, Differential Equations and Dynamical Systems, Springer-Verlag, New York, 1996.

[27] R. Plant, A Fitzhugh differential-difference equation modeling recurrent neural feedback, SIAM J. Appl. Math., 40 (1981), pp. 150-162.

[28] R. Qesmi, M. Ait Babram, And M. Hbid, Symbolic computation for center manifolds and normal forms of Bogdanov bifurcation in retarded functional differential equations, Nonlinear Analysis: Theory, Methods \& Applications, 66 (2007), pp. 2833-2851.

[29] B. Redmond, V. LeBlanc, And A. Longtin, Bifurcation analysis of a class of first order nonlinear delay-differential equations with reflectional symmetry, Physica D, 166 (2002), pp. 131-146.

[30] L. Shayer and S. Campbell, Stability, bifurcation and multistability in a system of two coupled neurons with multiple time delays, SIAM J. Appl. Math., 61 (2000), pp. $673-700$.

[31] J. Sieber and B. Krauskopf, Bifurcation analysis of an inverted pendulum with delayed feedback control near a triple-zero eigenvalue singularity, Nonlinearity, 17 (2004), pp. 85-103.

[32] G. StÉPÁn, Delay-differential equation models for machine tool chatter, in Dynamics and Chaos in Manufacturing Processes, F. Moon, ed., J. Wiley, New York, 1998, pp. 165191. 
[33] E. Stone And A. Askari, Nonlinear models of chatter in drilling processes, Dynamical Systems, 17 (2002), pp. 65-85.

[34] G. Süel, J. Garcia-Ojalvo, L. Liberman, and M. Elowitz, An excitable gene regulatory circuit induces transient cellular differentiation, Nature, 440 (2006), pp. 545550.

[35] H.-O. WALther, Bifurcation of periodic solutions with large periods for a delay differential equation, Annali di Matematica Pura ed Applicata, 185 (2006), pp. 1618-1891.

[36] W. Wischert, A. Wunderlin, A. Pelster, M. Olivier, and J. GroslamBERT, Delay-induced instabilities in nonlinear feedback systems, Phys. Rev. E, 49 (1994), pp. 203-219.

[37] D. Xiao And S. RuAn, Multiple bifurcations in a delayed predator-prey system with nonmonotonic functional response, J. Diff. Eqs., 176 (2001), pp. 464-510.

[38] P. YU AND Y. YUAN, The simplest normal forms associated with a triple zero eigenvalue of indices one and two, Nonlinear Analysis: Theory, Methods and Applications, 47 (2001), pp. 1105-1116.

[39] Y. YUAN AND J. WeI, Multiple bifurcation analysis in a neural network model with delays, Int. J. Bifurc. Chaos, 16 (2007), pp. 2903-2913.

[40] — Singularity analysis on a planar system with multiple delays, J. Dynamics and Differential Equations, 19 (2007), pp. 437-455. 